\title{
A STRONG TYPE OF REGULARITY FOR THE PWB SOLUTION OF THE DIRICHLET PROBLEM
}

\author{
D. H. ARMITAGE
}

\begin{abstract}
Let $H_{f}$ be the Perron-Wiener-Brelot solution of the Dirichlet problem for a resolutive function $f$ on the boundary $\partial \Omega$ of a bounded domain $\Omega$ in $E^{n}$. A point $y$ of $\partial \Omega$ will be called strongly regular if $H_{f}(x)$ $\rightarrow f(y)(x \rightarrow y)$ whenever $f$ is resolutive and continuous at $y$. Necessary and sufficient conditions for strong regularity are given.
\end{abstract}

1. Introduction. We use the notations and terminology of Helms [4], and we shall frequently make use of standard results in [4] without further notice. Throughout this note $\Omega$ will be a bounded domain (nonempty, connected, open set) in $E^{n}(n \geqslant 2)$ and $y$ will be a point of $\partial \Omega$. It is familiar that such a point $y$ is regular if and only if for each bounded, resolutive function $f$ on $\partial \Omega$, continuous at $y$,

$$
\lim H_{f}(x)=f(y){ }^{1}
$$

We shall say that $y$ is strongly regular if (1) holds for every resolutive function $f$ which is continuous at $y$. It is sometimes useful to know about strong regularity; for example, if every point of $\partial \Omega$ is strongly regular, then a continuous function $f$ on $\partial \Omega$ (possibly taking the values $\pm \infty$ ) has a classical Dirichlet solution $\left(=H_{f}\right)$ provided only that $\underline{H}_{f}>-\infty$ and $\bar{H}_{f}<+\infty$. In certain special cases (e.g., when $\Omega$ is a ball or a half-ball (Kuran [6])) one can show from explicit Poisson integral representations of $H_{f}$ that every point of $\partial \Omega$ is strongly regular. However, to the best of my knowledge, the problem of determining general conditions for strong regularity has not yet received attention.

For the purposes of comparison, we state the following theorem which is well known, at least implicitly. Its proof is omitted.

THEOREM A. A necessary and sufficient condition for the regularity of $y$ is that (1) holds for each nonnegative, real-valued, continuous function $f$ on $\partial \Omega$ with supp $f \subset \partial \Omega \sim B_{y, R}$ for some positive number $R$.

Received by the editors October 7, 1975.

AMS (MOS) subject classifications (1970). Primary 31B05, 31B20; Secondary 31A05, 31A25.

Key words and phrases. Dirichlet problem, regular boundary point.

1 Unless otherwise stated, 'lim' means ' $\lim _{x \rightarrow y}$ ' 
For the strong regularity of $y$, we need the condition of this theorem to hold, in a certain sense, uniformly in $f$. More precisely, we shall prove

THEOREM 1. Suppose that $z \in \Omega$. The following are equivalent:

(i) $y$ is strongly regular,

(ii) for each pair of positive numbers $R, \varepsilon$, there exists a positive number $r$ such that

$$
H_{f}(x) \leqslant \varepsilon H_{f}(z) \quad\left(x \in B_{y, r} \cap \Omega\right)
$$

for each nonnegative, real-valued continuous function $f$ with supp $f \subset \partial \Omega$ $\sim B_{y, R}$,

(iii) for each pair of positive numbers $R, \varepsilon$, there exists a positive number $r$ such that (2) holds for each nonnegative, resolutive function $f$ with supp $f \subset \partial \Omega$ $\sim B_{y, R}$.

A more geometrical condition for strong regularity is given by

THEOREM 2. If there exists a positive number such that $\Omega \cap B_{y, s}$ is a Lipschitz domain, then $y$ is stronly regular. In particular, if $\Omega$ is a Lipschitz domain, then every point of $\partial \Omega$ is strongly regular.

The proof depends on a result of Kemper [5].

Obviously, strong regularity implies regularity, and Theorems A and 1 lead one to suspect that regularity does not imply strong regularity. In fact, Brelot [1] has given an example of a domain in $E^{2}$ with a boundary point which is regular but not strongly regular. It is easy to show that his domain has a continuum of such boundary points.

2. Proof of Theorem 1. Since (iii) $\Rightarrow$ (ii) trivially, it is enough to show that (i) $\Rightarrow$ (iii) and (ii) $\Rightarrow$ (i).

(i) $\Rightarrow$ (iii) Suppose that $y$ is strongly regular and that $R>0$. Let $R$ be the vector space of resolutive functions $f$ on $\partial \Omega$ with supp $f \subset \partial \Omega \sim B_{y, R}$. We show first that there exist positive numbers $t, K$ such that $t<R$ and

$$
\left|H_{f}(x)\right| \leqslant K H_{|f|}(z) \quad\left(x \in \bar{B}_{y, t} \cap \Omega, f \in \Re\right) .
$$

Working by contradiction, we see that if (3) is false, then there exist a sequence $\left(x_{j}\right)$ in $\Omega$ such that $x_{j} \rightarrow y$ and a sequence $\left(f_{j}\right)$ in $R$ with the property

$$
\left|H_{f_{j}}\left(x_{j}\right)\right|\left(H_{\left|f_{j}\right|}(z)\right)^{-1} \rightarrow \infty \quad(j \rightarrow \infty) .
$$

With the norm \|\| , defined by

$$
\|f\|=H_{|f|}(z)=\int_{\partial \Omega}|f| d \mu_{z},
$$


$\Re=L^{1}\left(\mu_{z} \mid \partial \Omega \sim B_{y, R}\right)$ and is therefore a Banach space. (More properly, the set of $\mu_{z}$-equivalence classes in $R$ is a Banach space, but the distinction is unimportant here.)

For each $j$,

$$
f \leadsto H_{f}\left(x_{j}\right)=\int_{\partial \Omega} f d \mu_{x_{j}}
$$

is a linear operator on $\Re$, and, by a corollary of the Harnack inequalities (du Plessis [3, p. 47]), there is a real sequence $\left(k_{j}\right)$ such that

$$
\left|H_{f}\left(x_{j}\right)\right| \leqslant H_{|f|}\left(x_{j}\right) \leqslant k_{j} H_{|f|}(z)=k_{j}\|f\| \quad(j=1,2, \ldots, f \in \Re) .
$$

Since $y$ is strongly regular, $\lim _{j \rightarrow \infty} H_{f}\left(x_{j}\right)=f(y)=0(f \in \Re)$. The BanachSteinhaus theorem now gives the existence of a real number $K_{1}$ such that

$$
\left|H_{f}\left(x_{j}\right)\right| \leqslant K_{1}\|f\|=K_{1} H_{|f|}(z) \quad(j=1,2, \ldots, f \in \Re),
$$

which contradicts (4) and therefore proves (3).

Now let $\Omega^{\prime}=B_{y, t} \cap \Omega$ and define $\chi$ on $\partial \Omega^{\prime}$ by

$$
\chi=K \text { on } \partial \Omega^{\prime} \cap \Omega, \quad \chi=0 \text { on } \partial \Omega^{\prime} \cap \partial \Omega .
$$

Then $\chi$ is lower semicontinuous and bounded on $\partial \Omega^{\prime}$. It is therefore resolutive. Since $y$ is a strongly regular boundary point of $\Omega$, it is a regular boundary point of $\Omega$ and therefore a regular boundary point of $\Omega^{\prime}$. Hence, if $\varepsilon>0$, since $\chi$ is nonnegative and bounded on $\partial \Omega^{\prime}$ and is continuous at $y$, there exists a positive number $r$ such that $0 \leqslant H_{\chi}(x)<\varepsilon\left(x \in B_{y, r} \cap \Omega\right)$.

Suppose now that $f \in \mathscr{R}$ and $f \geqslant 0$. Define $f^{\prime}$ on $\partial \Omega^{\prime}$ by

$$
f^{\prime}=H_{f} \text { on } \partial \Omega^{\prime} \cap \Omega, \quad f^{\prime}=f=0 \text { on } \partial \Omega^{\prime} \cap \partial \Omega .
$$

Then $f^{\prime}$ is resolutive and $H_{f^{\prime}}=H_{f}$ in $\Omega^{\prime}$ (Brelot [2, Chapter 8, Property $\varepsilon$ ]). By (3), $f^{\prime} \leqslant H_{f}(z) \chi$ on $\partial \Omega^{\prime}$, so that $H_{f^{\prime}} \leqslant H_{f}(z) H_{\chi}$ in $\Omega^{\prime}$. Hence

$$
H_{f}(x)=H_{f^{\prime}}(x) \leqslant H_{f}(z) H_{\chi}(x) \leqslant \varepsilon H_{f}(z) \quad\left(x \in B_{y, r} \cap \Omega\right) .
$$

(ii) $\Rightarrow$ (i) Suppose that (ii) holds. Let $f$ be a resolutive function on $\partial \Omega$, continuous at $y$. We need to prove (1). Suppose for the moment that $|f(y)|<+\infty$. Then, since $H_{f}=H_{f-f(y)}+f(y)$, we may assume that $f(y)$ $=0$.

Suppose that $\varepsilon>0$. Then there exists a positive number $\delta$ such that $f<\varepsilon$ on $\bar{B}_{y, \delta} \cap \partial \Omega$. Let $u$ be a positive superharmonic element of $\mathfrak{U}_{f}$ (take $v$ $\in \mathfrak{U}_{f}$, then for some real $\lambda, 0<v+\lambda \in \mathfrak{U}_{f}$ ), and define $F$ on $\partial \Omega$ by

$$
F(w)=0 \quad\left(w \in \bar{B}_{y, \delta} \cap \partial \Omega\right), \quad F(w)=\liminf _{x \rightarrow w} u(x) \quad\left(w \in \partial \Omega \sim \bar{B}_{y, \delta}\right) .
$$

Then it is easy to check that $F$ is nonnegative and lower semicontinuous. Further, $f \leqslant \varepsilon+F$ on $\partial \Omega$, so that 


$$
\lim \sup H_{f}(x) \leqslant \varepsilon+\lim \sup \bar{H}_{F}(x) \text {. }
$$

Since $F$ is nonnegative and lower semicontinuous on $\partial \Omega$, there exists an increasing sequence $\left(g_{j}\right)$ of nonnegative, real-valued, continuous functions on $\partial \Omega$ such that $g_{j} \rightarrow F(j \rightarrow \infty)$. Hence, since $\mathfrak{U}_{F}$ contains a superharmonic element (e.g. u), $\lim _{j \rightarrow \infty} H_{g_{i}}=\bar{H}_{F}<+\infty$. The inequalities $0 \leqslant g_{j} \leqslant F$ imply that each $g_{j}$ vanishes on $\bar{B}_{y, \delta} \cap \partial \Omega$, whence, by (ii), there exists a positive number $r$ such that

$$
H_{g_{j}}(x) \leqslant \varepsilon H_{g_{j}}(z)\left(\bar{H}_{F}(z)+1\right)^{-1} \quad\left(x \in B_{y, r} \cap \Omega\right) .
$$

Letting $j \rightarrow \infty$, we find that $\bar{H}_{F}<\varepsilon$ in $B_{y, r} \cap \Omega$. Combining this result with (6) and remembering that $\varepsilon$ is arbitrary we obtain $\lim \sup H_{f}(x)=0$, and this, taken together with the corresponding result for $-f$, yields (1).

It remains to deal with the cases where $f(y)= \pm \infty$. Suppose that $f(y)$ $=+\infty$. Then for each positive integer $m, f \wedge m$ is resolutive and continuous at $y$, so that, by the case already dealt with, for each $m$,

$$
\lim \inf H_{f}(x) \geqslant \lim H_{f \wedge m}(x)=(f \wedge m)(y)=m,
$$

whence the result follows. The proof when $f(y)=-\infty$ is similar.

3. Proof of Theorem 2. A result of Kemper [5, Theorem 2.4] implies the following

Lemma. Suppose that $0<t<R<s$ and $\Omega \cap B_{y, s}$ is a Lipschitz domain. If $z \in \Omega \cap B_{y, t}$, then there is a real number $K$ such that for every nonnegative harmonic function $h$ in $\Omega \cap B_{y, s}$ which vanishes on $\partial \Omega \cap B_{y, R}, h(x) \leqslant K h(z)(x$ $\left.\in \bar{B}_{y, t} \cap \Omega\right)$.

To prove Theorem 2, we show that if its hypotheses are satisfied, then so also is condition (ii) of Theorem 1 .

We may suppose without loss of generality that $R<s$. Let $f$ be nonnegative, real-valued and continuous on $\partial \Omega$, and suppose that supp $f \subset \partial \Omega$ $\sim B_{y, R}$. Put $\omega=\Omega \cap B_{y, s}$ and define $\phi$ on $\partial \omega$ by

$$
\phi=H_{f} \text { on } \partial \omega \cap \Omega, \quad \phi=f \text { on } \partial \omega \cap \partial \Omega .
$$

Then $\phi$ is resolutive and $H_{\phi}=H_{f}$ in $\omega$. Further, $\phi$ is bounded on $\partial \omega$ and vanishes on $\partial \Omega \cap B_{y, R}$. Since $\omega$ is Lipschitz, every point of $\partial \omega$ is regular, by the Poincaré exterior cone criterion. Hence

$$
\lim _{x \rightarrow w} H_{f}(x)=\lim _{x \rightarrow w} H_{\phi}(x)=0 \quad\left(w \in \partial \Omega \cap B_{y, R}\right) .
$$

By the lemma, if $0<t<R$ and $z \in \Omega \cap B_{y, s}$, then there is a real number $K$ such that for all such functions $f$,

$$
H_{f}(x) \leqslant K H_{f}(z) \quad\left(x \in \bar{B}_{y, t} \cap \Omega\right) .
$$


If we put $\Omega^{\prime}=B_{y, t} \cap \Omega$, then by considering the function $\chi$ defined by (5) and repeating the argument given in $\S 2$, we can show that, if $\varepsilon>0$, there exists a positive number $r$ such that (2) holds for all such $f$.

\section{REFERENCES}

1. M. Brelot, Sur la mesure harmonique et le problème de Dirichlet, Bull. Sci. Math. (2) 69 (1945), 153-156. MR 7, 522.

2. Eléments de la théorie classique du potentiel, Centre de Documentation Universitaire, Paris, 1969.

3. N. du Plessis, An introduction to potential theory, Oliver and Boyd, Edinburgh, 1970.

4. L. L. Helms, Introduction to potential theory, Interscience, New York, 1969. MR 41 \#5638.

5. J. T. Kemper, $A$ boundary Harnack principle for Lipschitz domains and the principle of positive singularities, Comm. Pure Appl. Math. 25 (1972), 247-255. MR 45 \#2193.

6. Ü. Kuran, Harmonic majorizations in half-balls and half-spaces, Proc. London Math. Soc. (3) 21 (1970), 614-636.

Department of Pure Mathematics, Queen's University of Belfast, Belfast BT7 INN, NORTHERN IRELAND 